\title{
The Overlay of Minimization Diagrams in a Randomized Incremental Construction
}

\author{
Haim Kaplan • Edgar Ramos • Micha Sharir
}

Received: 30 September 2007 / Revised: 6 December 2010 / Accepted: 17 December 2010 /

Published online: 6 January 2011

(C) Springer Science+Business Media, LLC 2011

\begin{abstract}
In a randomized incremental construction of the minimization diagram of a collection of $n$ hyperplanes in $\mathbb{R}^{d}$, for $d \geq 2$, the hyperplanes are inserted one by one, in a random order, and the minimization diagram is updated after each insertion. We show that if we retain all the versions of the diagram, without removing any old feature that is now replaced by new features, the expected combinatorial complexity of the resulting overlay does not grow significantly. Specifically, this complexity is $O\left(n^{\lfloor d / 2\rfloor} \log n\right)$, for $d$ odd, and $O\left(n^{\lfloor d / 2\rfloor}\right)$, for $d$ even. The bound is asymptotically tight in the worst case for $d$ even, and we show that this is also the case for $d=3$. Several implications of this bound, mainly its relation to approximate halfspace range counting, are also discussed.
\end{abstract}

Keywords Randomized incremental construction · Minimization diagrams · Lower envelopes · Overlays · Hyperplanes · Arrangements · Voronoi diagrams

\footnotetext{
Work by Haim Kaplan was partially supported by Grant 975/06 from the Israel Science Foundation (ISF) and by Grant 2006/204 from the U.S.-Israel Binational Science Foundation. Work by Micha Sharir was partially supported by NSF Grants CCR-05-14079 and CCF-08-30272, by Grant 2006/194 from the U.S.-Israel Binational Science Foundation, by Grants 155/05 and 338/09 from the Israel Science Fund, and by the Hermann Minkowski-MINERVA Center for Geometry at Tel Aviv University.
}

H. Kaplan $(\varangle) \cdot$ M. Sharir

School of Computer Science, Tel Aviv University, Tel Aviv 69978, Israel

e-mail: haimk@post.tau.ac.il

M. Sharir

e-mail: michas@post.tau.ac.il

E. Ramos

Department of Computer Science, Universidad Nacional de Colombia, Medellín, Colombia e-mail: earamosn@unalmed.edu.co

M. Sharir

Courant Institute of Mathematical Sciences, New York University, New York, NY 10012, USA 


\section{Introduction}

\subsection{The Overlay of Minimization Diagrams}

Let $H$ be a set of $n$ non-vertical hyperplanes in $\mathbb{R}^{d}$. The lower envelope $\operatorname{Env}(H)$ of $H$ is the boundary of the intersection of the lower halfspaces bounded by the hyperplanes of $H$. Alternatively, regarding each hyperplane as the graph of a linear function of $x_{1}, \ldots, x_{d-1}$, Env $_{H}$ is the graph of the pointwise minimum of these functions. Env $_{H}$ is the boundary of a convex polyhedron with at most $n$ facets, so, by the Upper Bound Theorem (see [17]), its overall combinatorial complexity (the number of its faces of all dimensions) is $O\left(n^{\lfloor d / 2\rfloor}\right)$, where the constant of proportionality depends on $d$. This bound is asymptotically tight in the worst case.

The minimization diagram $\operatorname{Min}(H)$ of $H$ is the projection of $\operatorname{Env}(H)$ onto the hyperplane $x_{d}=0$. It is a convex subdivision of this space, consisting of at most $n$ full-dimensional cells, with a total of $O\left(n^{\lfloor d / 2\rfloor}\right)$ faces of all dimensions.

$\operatorname{Env}(H)$ and $\operatorname{Min}(H)$ can be constructed using a standard randomized incremental algorithm due to Clarkson and Shor [9]. In this approach, we draw a random permutation of $H$, and insert the hyperplanes of $H$ one by one in this order. During the process, we maintain the lower envelope and the minimization diagram of the set of hyperplanes inserted so far, and update these structures after each insertion. It is well known [9] that the expected number of faces, of all dimensions, of all the versions of the envelope, during the entire randomized process, is only $O\left(n^{\lfloor d / 2\rfloor}\right.$ ) (where expectation is with respect to the drawn random permutation). The algorithm itself is implemented using conflict lists which store, for certain features $f$ of the arrangement, the list of all hyperplanes not yet inserted, which lie (at least partially) below $f$, so the insertion of any of them "destroys" $f$. The expected running time of the algorithm is proportional to the expected overall size of these lists, which is shown to be $O\left(n^{\lfloor d / 2\rfloor}\right)$, for $d \geq 4$, and $O(n \log n)$, for $d=3$. See [7,9] for details.

Suppose now that we do not discard any transient face of the envelope, and retain all these faces, even though they are (partially or fully) eliminated by further insertions of hyperplanes. When projected onto the hyperplane $x_{d}=0$, we obtain an overlay of all versions of the minimization diagram of $H$. For a bad insertion order, the complexity of such an overlay can be rather large (as a matter of fact, even without overlaying the incremental versions, the overall number of faces created during the insertion process can be rather large in a bad insertion order). However, as we show in this paper, the expected complexity of the overlay is fairly small for a random insertion order. Specifically, we show that this expected complexity is $O\left(n^{\lfloor d / 2\rfloor} \log n\right)$, for $d$ odd, and $O\left(n^{\lfloor d / 2\rfloor}\right)$, for $d$ even. Furthermore, we show that this bound is worst-case tight for $d=3$ (it is clearly also worst-case tight for every even $d \geq 4$ ). We believe that the bound is tight for every odd $d$, but so far this remains only a conjecture.

In an earlier version of this paper [15], we have obtained this bound (namely, the bound $O(n \log n)$ ) only for $d=3$, using a more involved proof. The present version extends the result to all dimensions, and simplifies the proof.

Using duality, we obtain that the same bounds hold for the expected complexity of the overlay of all the faces of the normal (or Gaussian) diagram that are generated on the unit sphere $\mathbb{S}^{d-1}$ during a randomized incremental construction of the $d$-dimensional convex hull of a set of $n$ points in $\mathbb{R}^{d}$. 
Moreover, we recall that the Voronoi diagram of a set $P$ of $n$ points in $\mathbb{R}^{d}$ is the minimization diagram of a collection of $n$ hyperplanes in $\mathbb{R}^{d+1}$ (all tangent to the reflection of the standard paraboloid); see [11]. It follows that the expected complexity of the overlay of all the Voronoi faces that are generated during a randomized incremental construction of the Voronoi diagram of $P$ is $O\left(n^{\lceil d / 2\rceil} \log n\right)$, for $d$ even, and $O\left(n^{\lceil d / 2\rceil}\right)$, for $d$ odd. Similarly, the power diagram of a collection of $n$ balls in $\mathbb{R}^{d}$ is the minimization diagram of a corresponding collection of $n$ hyperplanes in $\mathbb{R}^{d+1}$ (see, e.g., Boissonnat and Yvinec [8]), so a similar statement holds for the overlay of the faces of a power diagram during a randomized incremental construction thereof.

\subsection{Motivation and Background}

The overlay of the minimization diagrams, as defined above, has the following useful property. Denote by $\pi=\left(h_{1}, h_{2}, \ldots, h_{n}\right)$ the random insertion permutation, and let $f$ be a face of the overlay. Then there is a fixed subsequence $\pi_{f}=\left(h_{i_{1}}, h_{i_{2}}, \ldots, h_{i_{k}}\right)$ of $\pi$, so that, for every $x \in f$, the hyperplanes in $\pi_{f}$ are the prefix minima of the sequence $\left(h_{1}(x), h_{2}(x), \ldots, h_{n}(x)\right)$; that is, $\pi_{f}$ consists of exactly those hyperplanes $h_{j}$ for which $h_{j}(x) \leq h_{i}(x)$ for each $i<j$ and for each $x \in f$. Since $\pi$ is a random permutation, the expected size of $\pi_{f}$ is only $O(\log n)$. (This is easily seen to hold for all faces $f$. This follows from the fact that $\left(h_{1}(x), h_{2}(x), \ldots, h_{n}(x)\right)$ is a random permutation of these $n$ real numbers for every $x$ for which these numbers are distinct.) Hence, for any query point $x \in \mathbb{R}^{d-1}$, locating the face $f$ of the overlay which contains $x$ gives us the sequence of prefix minima of $\pi$ at $x$. Thus, after finding $f$, we can, in additional expected $O(\log n)$ time, answer queries of the following form: Given a point $w \in \mathbb{R}^{d}$, find the first hyperplane of $H$ (according to the order of $\pi$ ) that passes below $w$.

Answering queries of this form is needed when applying Cohen's technique [10] for approximate halfspace range counting. That is, given a set $P$ of $n$ points in $\mathbb{R}^{d}$, and a parameter $\varepsilon>0$, we want to preprocess $P$ into a data structure, so that, given a query halfspace $H$, we can compute an approximate count $M$ that satisfies

$$
(1-\varepsilon)|P \cap H| \leq M \leq(1+\varepsilon)|P \cap H| .
$$

Cohen's technique is a general method that estimates the number of data objects in a given range $H$ as follows. One assigns to each data object, independently, a random weight, drawn from an exponential distribution with density $e^{-x}$, sorts the objects by their weights into a random permutation, and then finds the minimum rank in that permutation of the objects in the query range $H$. One then repeats this experiment $O\left(\frac{1}{\varepsilon^{2}} \log n\right)$ times, computes the average $\mu$ of the weights of the minimum elements, and approximates $|P \cap H|$ by $1 / \mu$. (Cohen [10] also proposes several other estimators that have similar properties.) As shown in [10], this approximate count lies, with high probability, within relative error $\varepsilon$ of $|P \cap H|$, as in (1). See [10] for more details.

To apply this machinery for approximate halfspace range counting in $\mathbb{R}^{d}$, we need to solve the following problem: Given a set $P$ of $n$ points in $\mathbb{R}^{d}$ in general position, ${ }^{1}$

\footnotetext{
${ }^{1}$ To simplify the presentation, we assume throughout the paper that the data objects (points or hyperplanes) are in general position, in the sense discussed, e.g., in [7, p. 9,122].
} 
and a random permutation $\pi$ of $P$ (it is easily verified that the sorted order of the points of $P$ according to their randomly drawn weights is indeed a random permutation with probability 1 ; see [10]), construct a data structure that can answer efficiently halfspace minimum range queries of the form: Given a query halfspace $H$, find the point of $p \in P \cap H$ of minimum rank in $\pi$ (i.e., with the minimum value of $\pi(p)$ ). This can be solved by dualizing the problem, turning $P$ into a collection of $n$ nonvertical hyperplanes, and mapping each query halfspace $H$ to a point (dual to the hyperplane bounding the range), so that we want to find the minimum-rank hyperplane passing, say, below the query point. As explained above, this can be done by locating the projection $w^{*}$ of the query point $w$ onto $x_{d}=0$ in the overlay of the minimization diagrams, thereby obtaining the sequence of prefix minima of $\pi$ at $w^{*}$, which can then be searched to find the desired minimum-rank hyperplane below $w$.

In an earlier version of the paper [15], which has catered only to the 3-dimensional case, we have presented an algorithm that implements Cohen's technique by constructing the overlay along the lines described above. For the halfspace minimum range searching problem, the algorithm requires $O(n \log n)$ expected storage and preprocessing, and answers a query in $O(\log n)$ expected time; the storage and preprocessing bounds are derived from our bound on the overlay complexity, as stated above. While attempting to extend this technique to higher dimensions, we discovered that constructing the overlay is (a) somewhat inefficient, and (b) not really required. In a companion paper [14], we present a method for answering halfspace range minimum queries using standard range searching techniques (that is, cuttings). These techniques yield data structures with expected storage $O\left(n^{\lfloor d / 2\rfloor}(\log \log n)^{O(1)} / \log \lfloor d / 2\rfloor n\right)$, while a query still takes $O(\log n)$ expected time.

We also note that recently Afshani and Chan [1] have applied our overlay bound, in three dimensions, to obtain an optimal solution for the approximate halfspace range counting problem, for the 3 -dimensional case, which requires (ignoring the dependence on the approximation factor $\varepsilon) O(n)$ expected storage, $O(n \log n)$ expected preprocessing time, and answers an approximate halfspace counting query in $O(\log (n / k))$ expected time, where $k$ is the actual count. Unlike the approach based on Cohen's technique, the algorithm of [1] uses a variety of random sources, not just a single random permutation of the input.

Finally, approximate halfspace range counting has by now several competing solutions, which have been developed during the past several years, due to Aronov and Har-Peled [4], Aronov and Sharir [6] (see also [5]), Afshani and Chan [1] (only in three dimensions), and, as noted above, to Kaplan et al. [14].

Our new bound on the complexity of the overlay of diagrams of these kinds is related to the work of Guibas et al. [12] on randomized incremental construction of planar Voronoi diagrams. They build a data structure storing the Voronoi regions that are generated during a randomized incremental construction of the diagram, such that one can efficiently obtain the entire sequence of Voronoi regions that contain a query point; that is, the sequence of nearest neighbors to the query point, during the incremental construction. The time it takes to answer a query, using their structure, is $O\left(\log ^{2} n\right)$. Guibas et al. [12] pose it as an open problem to improve the query time to $O(\log n)$. Our overlay bound in three dimensions provides a partial solution to this 
problem. Namely, it leads to an alternative structure that can answer a point location query of this kind in $O(\log n)$ time, at the cost of increasing its expected storage size to $O(n \log n)$; see the earlier version [15] for details. In [14], we show how to answer queries of these form without using the overlay, so that only linear space is required.

Another result related to our complexity bound for the overlay of minimization diagrams is due to Agarwal et al. [3], who have developed a kinetic binary space partitioning (BSP) technique for a set of moving interior-disjoint segments in the plane. To obtain this result, they consider the overlay of the vertical decompositions of prefixes of a random insertion sequence of the segments, and give a very simple proof that the expected complexity of this overlay is $O(n \log n)$. Let $\sigma_{1}, \ldots, \sigma_{k}$ be the segments that intersect the vertical ray $\rho$ emanating upwards from an endpoint of a segment $s$, in increasing order of their $y$-coordinates. Then $\sigma_{i}$ crosses (a portion of) $\rho$ in the overlay if and only if $s$ is inserted before $\sigma_{1}, \ldots, \sigma_{i}$. Since we add the segments in random order, the probability that $\rho$ crosses $\sigma_{i}$ is $1 /(i+1)$. Therefore, the expected number of segments crossing $\rho$ is at most $\sum_{i=1}^{n} 1 /(i+1)=O(\log n)$, and repeating it for each segment endpoint and each incident vertical ray yields the asserted bound.

However, in our case, a crossing in the overlay of, say, Voronoi regions in $\mathbb{R}^{2}$, is determined by four of the inserted points, whereas a crossing of the kind studied by [3] is determined by only two segments. This tends to make the analysis in our case more intricate, and even more so in higher dimensions.

Agarwal, Ericksen, and Guibas [2], still in the context of developing kinetic BSP structures, extend the result of Agarwal et al. [3] to intersecting segments. In this case, the bound on the complexity of the overlay of the vertical decomposition of the segments is $O(n \log n+k)$, where $k$ is the number of segment intersections. In three dimensions, they prove a bound of $O\left(n \log ^{2} n+k\right)$ on the complexity of the overlay of the vertical decomposition of $n$ triangles, where $k$ is the number of intersections between the projections of the edges of the triangles onto the $x y$-plane.

Finally, we mention the work of Har-Peled [13], who has studied the randomized incremental construction of a $(1 / r)$-cutting of an arrangement of $n$ lines in the plane, using an approach that does not delete old features of the cutting as new lines are inserted. Har-Peled shows that this does not affect the asymptotic complexity of the resulting cutting, which remains $O\left(r^{2}\right)$.

\section{Overlaying Incremental Minimization Diagrams}

Before presenting our bounds, let us illustrate the scenario in the 3-dimensional case. We thus have a set $H$ of $n$ non-vertical planes in $\mathbb{R}^{3}$, and a random permutation $\pi$ thereof. Let $H_{i}$ denote the subset of the first $i$ elements in $\pi$, for $i=1,2, \ldots, n$. Let $\operatorname{Env}\left(H_{i}\right)$ and $\operatorname{Min}\left(H_{i}\right)$ denote respectively the lower envelope and the minimization diagram of $H_{i}$. We insert the planes of $H$ one at a time, in their $\pi$-order. After inserting the $i$ th plane, we construct $\operatorname{Env}\left(H_{i}\right)$ and $\operatorname{Min}\left(H_{i}\right)$ from $\operatorname{Env}\left(H_{i-1}\right)$ and $\operatorname{Min}\left(H_{i-1}\right)$. During the incremental construction, we collect the edges of the newly generated faces of all the versions $\operatorname{Min}\left(H_{i}\right)$ of the minimization diagram into a "global" set $G$. The overlay of the minimization diagrams, which we denote as 
$\operatorname{Ov}(H, \pi)$, is thus the final arrangement $\mathcal{A}(G)$; clearly, each face $f$ of $\mathcal{A}(G)$ is contained in a single face of each of the minimization diagrams $\operatorname{Min}\left(H_{i}\right)$, so knowing the face of $\mathcal{A}(G)$ that contains a query point $q$ gives us the sequence of the prefix minima of $\pi$ at $q$.

An illustration of the process is given in Figs. 1 and 2, for the special case of Voronoi diagrams. (Recall that the Voronoi diagram of a set $P$ of $n$ points in the plane can be interpreted as the minimization diagram of $n$ dual planes in 3-space.) Figure 1 illustrates the evolution of the Voronoi diagram as each of the original points (equivalently, dual planes) is inserted. Figure 1 also depicts a query disk $D$, which in dual 3-space becomes a point $D^{*}$. Each time the center of the disk "moves" to a new Voronoi face, its nearest neighbor in the prefix set changes (and gets closer) until this neighbor enters the disk. In dual 3-space, the sequence of nearest neighbors map
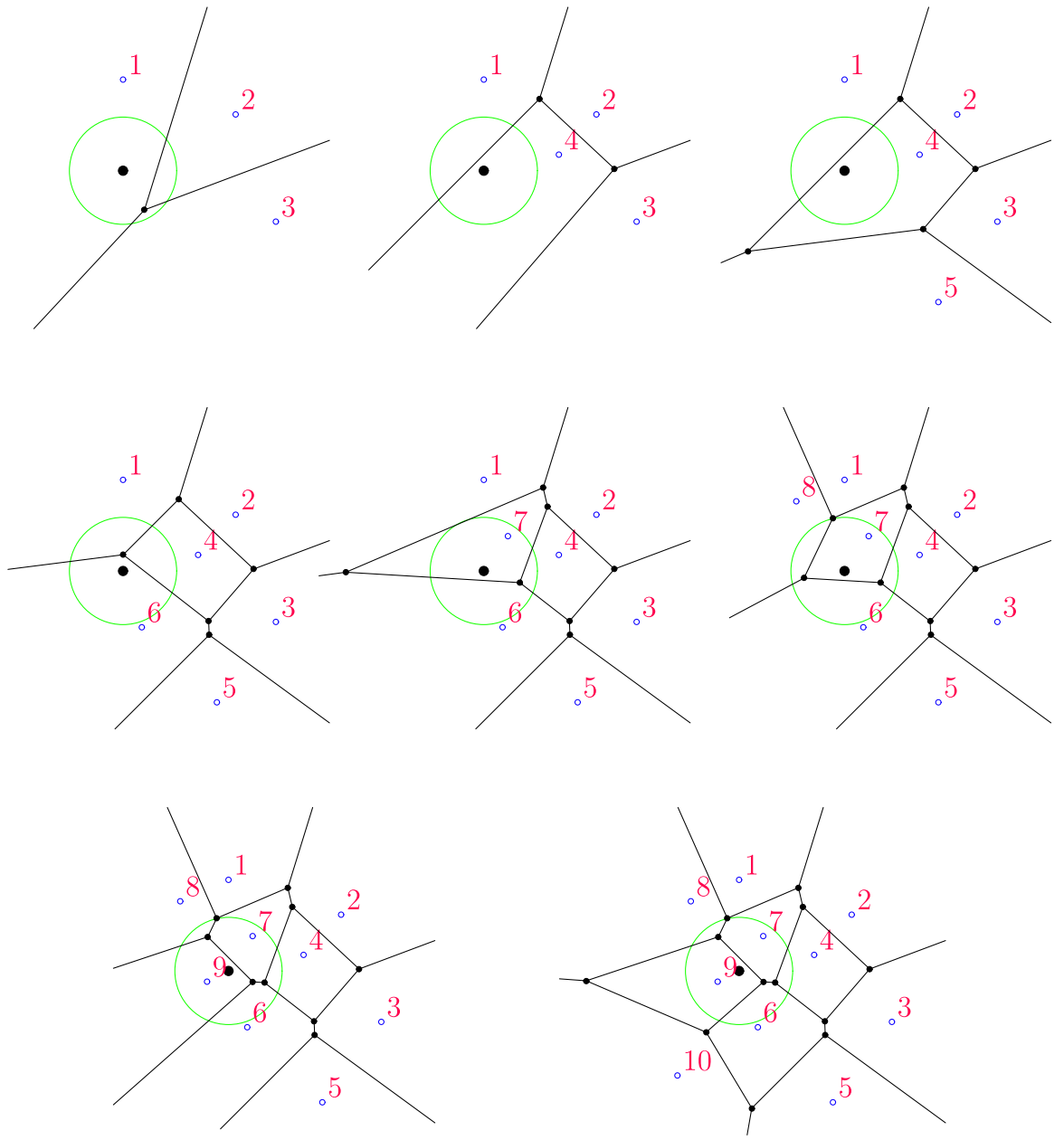

Fig. 1 Randomized incremental construction of the Voronoi diagram of 10 points in the plane 
Fig. 2 The overlay arrangement $\mathcal{A}(G)$ for the incremental Voronoi diagram of the point set in Fig. 1. As above, the sites are drawn as hollow circles and are labeled by their ranks in $\pi$. The query disk from the preceding figure is also shown. For the (triangular) face $f$ of the overlay containing its center, the sequence of prefix minima at $f$ is $(1,4,6,7,9)$

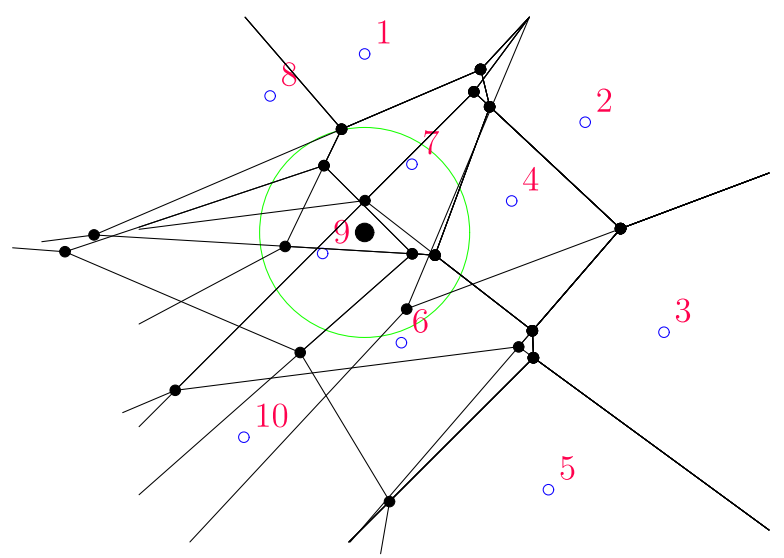

to the sequence of prefix minima at the $x y$-projection of $D^{*}$ (which is the center of the disk), and the first nearest neighbor to enter the disk corresponds to the first plane to pass below $D^{*}$. The first point to enter $D$ is point 7 in the figure, and the prefix minima sequence at (which is the sequence of nearest neighbors to) the center of the disk is $(1,4,6,7,9)$.

The overlay of the minimization diagrams is depicted in Fig. 2.

\section{The Complexity of the Overlay of Minimization Diagrams}

In this section, we analyze the complexity of the overlay of incrementally constructed minimization diagrams of a set of $n$ hyperplanes in $\mathbb{R}^{d}$. Our main result is:

Theorem 3.1 The expected combinatorial complexity of the overlay of the minimization diagrams that arise during a randomized incremental construction of the lower envelope of $n$ hyperplanes in $\mathbb{R}^{d}$, for $d \geq 2$, is $O\left(n^{\lfloor d / 2\rfloor}\right)$, for $d$ even, and $O\left(n^{\lfloor d / 2\rfloor} \log n\right)$, for $d$ odd. The bounds for $d$ even and for $d=3$ are tight in the worst case.

Proof For $d \geq 2$ and $n \geq 1$, define $F_{d}(n)$ as follows:

$$
F_{d}(n)= \begin{cases}C_{d} n^{\lfloor d / 2\rfloor}, & d \text { is even, } \\ C_{d} n^{\lfloor d / 2\rfloor} \log n, & d \text { is odd },\end{cases}
$$

with appropriate constants of proportionality $C_{d}$ that depend on $d$. We prove, by induction on $d$, that $F_{d}(n)$ is an upper bound on the complexity of the overlay of the minimization diagrams that arise during a randomized incremental construction of the lower envelope of $n$ hyperplanes in $\mathbb{R}^{d}$. (The choice of the constants $C_{d}$ will follow from the inductive proof.)

The basis of the induction is $d=2$. In this case, the overlay is a subdivision of the line, and all its vertices are projections of the vertices of the envelopes $\operatorname{Env}\left(H_{i}\right)$. 
Since each newly inserted line creates at most two new envelope vertices, the overall number of vertices is at most $2 n=2 n^{\lfloor d / 2\rfloor}$, and the claim follows (with $C_{2}=2$ ).

Let $d \geq 3$ and suppose that the bound holds for any dimension $d^{\prime}<d$. It is easily seen that, assuming general position of the input hyperplanes, the complexity of the overlay is proportional to the number of its vertices. (Indeed, most faces of the overlay have at least one incident vertex, and each vertex is incident to only $O(1)$ faces, with a constant of proportionality depending only on $d$, as follows from our general position assumption. Faces with no vertices are unbounded, and can be "charged" to dummy incident vertices at infinity, whose number can be bounded by much the same way as the number of real vertices.) Note that the general position assumption involves no loss of generality, since the complexity of the overlay can only grow when we slightly perturb a degenerate configuration into general position.

A vertex $v$ of the overlay $\operatorname{Ov}(H, \pi)$ is the intersection of several faces $f_{1}^{*}, \ldots, f_{k}^{*}$ of various dimensions of different minimization diagrams, whose sum of codimensions in $\mathbb{R}^{d-1}$ is $d-1$, and each co-dimension is at least 1 . Let $f_{i}$ denote the face of the corresponding lower envelope, whose orthogonal projection onto $\mathbb{R}^{d-1}$ is $f_{i}^{*}$, for $i=1, \ldots, k$. We assume, without loss of generality, that the faces $f_{1}, \ldots, f_{k}$ are generated in this order during the incremental construction. We ignore the case $k=1$, since then $v$ is a vertex of a single minimization diagram, and the expected overall number of such vertices, over the entire incremental process, is $O\left(n^{\lfloor d / 2\rfloor}\right)$, as follows, e.g., from the standard technique of Clarkson and Shor [9].

Note that a fixed vertex $v$ of the overlay can be generated by different insertion orders and by different sequences of faces $f_{1}, \ldots, f_{k}$. Each such face $f_{i}$ depends, in addition to the hyperplanes that contain it, on the hyperplanes that delimit it (and pass above it). When we change the insertion order of the hyperplanes, faces with the same containing hyperplanes, but with different delimiting hyperplanes may be formed, so that their vertical projections still intersect at $v$.

In what follows, we assume that each face of dimension up to $d-2$ (recall that we only deal with faces whose co-dimension is at least 1) of the lower envelope $\operatorname{Env}(H)$, of any subset $H$ of the hyperplanes, is triangulated into simplices, using, e.g., the bottom-vertex triangulation technique (see, e.g., [16]). Any such simplex is determined in terms of at most some constant number $c=c(d)$ of hyperplanes. (For example, in $d=4$ dimensions, we need up to 7 hyperplanes to define a triangle $\Delta$ : two hyperplanes define the 2-plane containing $\Delta$, three additional ones define a "real" edge of $\Delta$ (an original edge of the 2-face containing $\Delta$, including its endpoints), and two more hyperplanes define the bottom vertex of that 2-face.) Let $g$ be a simplex (of any dimension) in the triangulation of a face $f$ of $\operatorname{Env}(H)$, for some subset $H$. Let $W(g)$ be the vertical prism consisting of all the points in $\mathbb{R}^{d}$ that lie vertically strictly below $g$ in the $x_{d}$-direction. Let $C(g)$ denote the conflict list of $g$; it is the subset of hyperplanes $h \in H$ that cross $W(g)$; that is, each of them passes, at least partially, below $g$. Put $w(g)=|C(g)|$ and refer to it as the weight of $g$.

Let now $v$ be a vertex of the overlay $\operatorname{Ov}(H, \pi)$, formed by the intersection of $k \geq 2$ faces of the evolving minimization diagram. Then $v$ lies in the projection of some simplex $g$ in the bottom-vertex triangulation of the highest face $f_{1}$ (among those whose projections intersect at $v$ ). As discussed above, $v$ does not uniquely determine $f_{1}$, and, for similar reasons, $g$ is also not necessarily uniquely defined. 
For $v$ to appear in the overlay, there must exist such a simplex $g$, of dimension $d(g)$, that arises in the bottom-vertex triangulation of some version of the envelope, such that $v$ appears in the overlay of $\operatorname{Env}(C(g))$ in the $(d(g)+1)$-dimensional affine hull of $W(g)$. (Indeed, since the face $f_{1}$ is the highest among the faces whose vertical projection contains $v$, all the hyperplanes which contain the other faces $f_{2}, \ldots, f_{k}$ must belong to $C(g)$.)

We fix a simplex $g$, as above, and condition the probability of the event that $v$ appears in the overlay, on the event that $g$ shows up in the bottom-vertex triangulation of the envelope during the construction. It follows from the preceding discussion that this conditional probability is equal to the probability that $v$ arises during a randomized incremental construction of $\operatorname{Env}(C(g))$, in the $(d(g)+1)$-dimensional affine hull of $W(g)$. (It is important to realize that restricting the problem to $C(g)$ may affect the other faces whose projections meet at $v$ because their delimiting hyperplanes need not belong to $C(g)$. Nevertheless, the hyperplanes containing these faces are all in $C(g)$; using this fact, the equality between the probabilities follows.) By the induction hypothesis, the expected number of such vertices (conditioned on $g$ appearing on the envelope) is at most $F_{d(g)+1}(w(g))$. (For this to work, we need the following easy but crucial property: Conditioned on $g$ being created, the order in which the elements of $C(g)$ are inserted is a random permutation.) Therefore, the expected complexity of the overlay of the minimization diagrams is at most

$$
\sum_{g} \operatorname{Prob}[g \text { is generated }] \cdot F_{d(g)+1}(w(g)),
$$

where the sum is over all possible simplices $g$ that can show up in the bottom-vertex triangulation of the lower envelope of up to $c$ hyperplanes of $H$.

For simplicity of exposition, we bound the sum in (2), assuming that all simplices $g$ are determined by exactly $c$ hyperplanes. (For the complete analysis, we consider each possible number $c^{\prime} \leq c$ of hyperplanes that determine $g$, repeat the argument given below for each $c^{\prime}$, and add up the bounds, all of which will have the same asymptotic form.) The simplex $g$ is generated if all $c$ hyperplanes defining it appear in the permutation before any of the $w(g)$ hyperplanes in its conflict list. Thus the probability that $g$ is generated is

$$
\frac{c ! w(g) !}{(c+w(g)) !}=O\left(\frac{1}{(w(g)+1)^{c}}\right) .
$$

Let $N_{w}$ denote the number of simplices $g$ of dimension at most $d-2$ and weight $w=w(g)$ (which are defined by $c$ hyperplanes). Then, since $F_{d(g)+1}(w(g)) \leq$ $F_{d-1}(w(g))$, the expected number of vertices in question (given in (2)) is

$$
O\left(\sum_{w \geq 0} \frac{N_{w} F_{d-1}(w)}{(w+1)^{c}}\right)
$$


Let $N_{\leq w}$ denote the number of simplices, as above, of weight at most $w$. Then the sum can be rearranged as

$$
O\left(\sum_{w=0}^{n-c-1} N_{\leq w}\left[\frac{F_{d-1}(w)}{(w+1)^{c}}-\frac{F_{d-1}(w+1)}{(w+2)^{c}}\right]+\frac{N_{\leq n-c} F_{d-1}(n-c)}{(n-c+1)^{c}}\right),
$$

The Clarkson-Shor bound [9] implies that

$$
N_{\leq w}=O\left((w+1)^{c} N_{0}(n /(w+1))\right)=O\left((w+1)^{c-\lfloor d / 2\rfloor} n^{\lfloor d / 2\rfloor}\right) .
$$

By definition, we have

$$
\frac{F_{d-1}(w)}{(w+1)^{c}}-\frac{F_{d-1}(w+1)}{(w+2)^{c}}= \begin{cases}O\left((w+1)^{\lfloor(d-1) / 2\rfloor-c-1}\right), & \text { for } d \text { odd } \\ O\left((w+1)^{\lfloor(d-1) / 2\rfloor-c-1} \log (w+1)\right), & \text { for } d \text { even }\end{cases}
$$

(We assume here that $c \geq\lfloor(d-1) / 2\rfloor$; otherwise the sum is negative, and only the last term is positive.) We substitute this into (3), observe that the last term of that equation is $O\left(F_{d-1}(n)\right)$, and consequently get that, for even $d$, the expected number of vertices of the overlay is

$$
\begin{aligned}
& O\left(\sum_{w \geq 0}(w+1)^{c-\lfloor d / 2\rfloor} n^{\lfloor d / 2\rfloor} \cdot(w+1)^{\lfloor(d-1) / 2\rfloor-c-1} \log (w+1)\right) \\
& =O\left(n^{\lfloor d / 2\rfloor}\right) \cdot \sum_{w \geq 0}(w+1)^{\lfloor(d-1) / 2\rfloor-\lfloor d / 2\rfloor-1} \log (w+1) .
\end{aligned}
$$

For even $d$, the latter sum equals to $\sum_{w \geq 0}(w+1)^{-2} \log (w+1)=O(1)$. If $d$ is odd then the expected number of vertices of the overlay is bounded by a similar expression as in (4), except that the factor $\log (w+1)$ does not appear, and the sum is then $\sum_{w \geq 0}(w+1)^{-1}=O(\log (w+1))=O(\log n)$. Recall that we have ignored overlay vertices that are the projections of vertices of the envelope (although such vertices did show up in the inductive bounds). The expected number of these vertices is $O\left(n^{\lfloor d / 2\rfloor}\right)$, so including them in the overall count does not affect the asymptotic bounds. This establishes the induction step, with an appropriate choice of the constants of proportionality $C_{d}$, and thus completes the proof of the upper bound in the theorem.

\subsection{A Lower Bound for the Overlay Complexity in $\mathbb{R}^{3}$}

The upper bound is clearly worst-case tight for $d$ even, since it (asymptotically) coincides with the tight worst-case bound for the complexity of just the final version of the envelope. To complete the proof of Theorem 3.1, we next provide a construction where the expected complexity of the overlay is $\Theta(n \log n)$ in $\mathbb{R}^{3}$.

Consider a set $H$ of $2(n+1)$ planes, partitioned into two sets $U$ and $Q$. The set $U$ consists of $n+1$ planes $\left\{u_{1}, \ldots u_{n+1}\right\}$, all tangent from above to a sufficiently small cylinder around the $x$-axis, so that the directions of their normals span a sufficiently 
Fig. 3 The lower bound construction in $\mathbb{R}^{3}$ : a side view

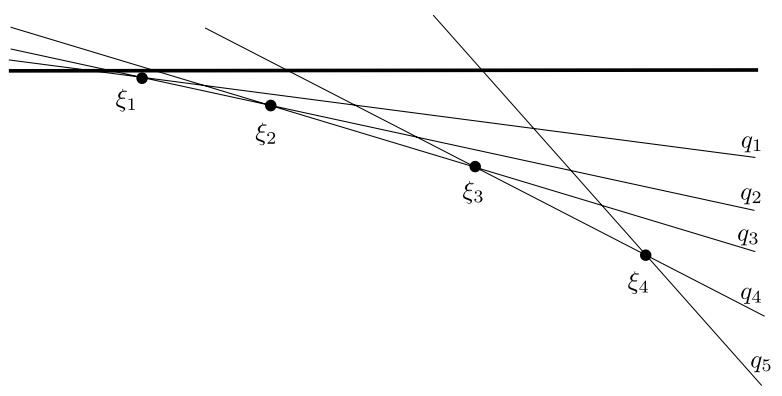

small angle near the positive $z$-direction. The set $U$ has the property that, for any subset $U^{\prime} \subseteq U$, all the planes in $U^{\prime}$ appear on the lower envelope of $U^{\prime}$, which consists of $\left|U^{\prime}\right|$ strips parallel to the $x$-axis, separated by $\left|U^{\prime}\right|-1$ lines parallel and very close to the $x$-axis.

The set $Q$ consists of $n+1$ planes $\left\{q_{1}, \ldots q_{n+1}\right\}$, all parallel to the $y$-axis. It is simpler to present the construction of $Q$ by describing the cross-section of their arrangement within the $x z$-plane, which is depicted in Fig. 3. The line representing $q_{1}$ emanates from the origin and has a slightly negative slope. Suppose that the lines representing $q_{1}, \ldots, q_{i}$ have already been constructed. We then construct the line representing $q_{i+1}$ so that it emanates from a point on the $x$-axis that lies to the right of the intersection point of $q_{i-1}$ and $q_{i}$ (or of the origin, for $i=1$ ), and its slope is slightly smaller (more negative) than that of $q_{i}$.

Fix $u \in U$, and consider the event $A_{u, k}$ that when $u$ is inserted $k$ is the smallest index of the planes in $Q$ that have already been inserted. In that case, the projection of the facet generated by $u$ will intersect the projections of all the facets generated by the planes $q_{i}$ of "larger slope", i.e., for $1 \leq i<k$. Each of these intersections will contribute (at least) a constant number of vertices to the overlay.

The probability of $A_{u, k}$ is $1 /(k(k+1))$, since in the set $\left\{u, q_{1}, \ldots, q_{k}\right\}$ the plane $q_{k}$ needs to be inserted first and the plane $u$ second. The contribution of $A_{u, k}$ to the complexity of the overlay is $\Omega(k)$, so its expected contribution is $\Omega(1 / k)$. Summing this expectation over all $k$ and $u$ gives a total expected contribution of $\Omega(n \log n)$, which establishes the asserted lower bound.

Remark We conjecture, and leave it as an open problem to show, that the upper bounds of Theorem 3.1 are worst-case tight for every (odd) dimension $d$.

Acknowledgements We would like to thank the two anonymous referees for their helpful suggestions and comments. In particular, the proof of the lower bound in three dimensions was considerably simplified due to their comments.

\section{References}

1. Afshani, P., Chan, T.M.: On approximate range counting and depth. Discrete Comput. Geom. 42, 3-21 (2009)

2. Agarwal, P.K., Erickson, J., Guibas, L.: Kinetic binary space partitions for intersecting segments and disjoint triangles. In: Proc. 9th Annu. ACM-SIAM Sympos. Discrete Algo, pp. 107-116 (1998) 
3. Agarwal, P.K., Guibas, L.J., Murali, T.M., Vitter, J.S.: Cylindrical static and kinetic binary space partitions. Comput. Geom. Theory Appl. 16, 103-127 (2000)

4. Aronov, B., Har-Peled, S.: On approximating the depth and related problems. SIAM J. Comput. 38, 899-921 (2008)

5. Aronov, B., Har-Peled, S., Sharir, M.: On approximate halfspace range counting and relative epsilon approximations. In: Proc. 23rd Annu. ACM Sympos. on Comput. Geom., pp. 327-336 (2007)

6. Aronov, B., Sharir, M.: Approximate halfspace range counting. SIAM J. Comput. 39, 2704-2725 (2010)

7. de Berg, M., van Kreveld, M., Overmars, M., Schwarzkopf, O.: Computational Geometry: Algorithms and Applications, 2nd edn. Springer, Heidelberg (2000)

8. Boissonnat, J.-D., Yvinec, M.: Algorithmic Geometry (English edn.). Cambridge University Press, Cambridge (1998)

9. Clarkson, K., Shor, P.: Applications of random sampling in computational geometry, II. Discrete Comput. Geom. 4, 387-421 (1989)

10. Cohen, E.: Size-estimation framework with applications to transitive closure and reachability. J. Comput. Syst. Sci. 55, 441-453 (1997)

11. Edelsbrunner, H., Seidel, R.: Voronoi diagrams and arrangements. Discrete Comput. Geom. 1, 25-44 (1986)

12. Guibas, L., Knuth, D.E., Sharir, M.: Randomized incremental construction of Voronoi and Delaunay diagrams. Algorithmica 7, 381-413 (1992)

13. Har-Peled, S.: Constructing planar cuttings in theory and practice. SIAM J. Comput. 29, 2016-2039 (2000)

14. Kaplan, H., Ramos, E., Sharir, M.: Range minima queries with respect to a random permutation, and approximate range counting. Discrete Comput. Geom. (in press); published online November 11, 2010. doi:10.1007/s00454-010-9308-6

15. Kaplan, H., Sharir, M.: Randomized incremental constructions of three-dimensional convex hulls and planar Voronoi diagrams, and approximate range counting. In: Proc. 17th Annu. ACM-SIAM Sympos. Discrete Algo, pp. 484-493 (2006)

16. Sharir, M., Agarwal, P.K.: Davenport-Schinzel Sequences and Their Geometric Applications. Cambridge University Press, New York (1995)

17. Ziegler, G.: Lectures on Polytopes, Graduate Texts in Math., vol. 152. Springer, New York (1995) 